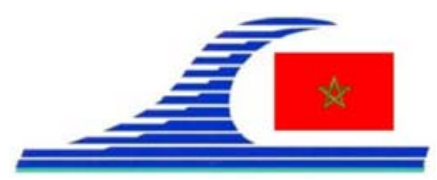

Conférence Méditerranéenne Côtière et Maritime EDITION 2, TANGER, MAROC (2011)

Coastal and Maritime Mediterranean Conference

Disponible en ligne - http://www.paralia.fr - Available online

\title{
Aspects de la modélisation numérique des tsunamis
}

\section{Houssam Eddine TOUHAMI ${ }^{1}$, Mouhamed Chérif KHELLAF ${ }^{1}$}

1. Université des Sciences et de la Technologie Houari Boumedien USTHB, Faculté de génie civil, laboratoire LEGHYD,

BP 32 El Alia Bab-Ezzouar 16111 Alger. Algérie.

touhami.houssam.e@hotmail.com;mckhellaf@yahoo.fr

\section{Résumé :}

La présente étude constitue une modélisation numérique d'une vague solitaire de tsunami dans le cas d'une dimension, en utilisant les équations de Boussinesq modifiées par WEI et al. (1995), et le code de calcul COULWAVE. Pour cela, nous avons repris en simulation numérique le cas étudié expérimentalement par SYNOLAKIS (1987). Le travail a été fait sur une plage plane uniforme de pente 1:19.85, pour un rapport entre amplitude de vague solitaire et profondeur d'eau égale à 0.0185 . Et enfin, nous avons comparé les résultats obtenus avec ceux expérimentaux de Synolakis.

\section{Mots-clés :}

Tsunami - Equations de Boussinesq - Simulation numérique - COULWAVE

\section{Introduction}

Le problème de la propagation des tsunamis est un cas particulier de la propagation des ondes. Toutes les équations utilisées pour la modélisation des vagues dérivent des équations de Navier-Stokes, néanmoins il existe plusieurs modèles de tsunami qui diffèrent dans :

a) la forme de l'onde : l'onde solitaire (SYNOLAKIS, 1987) et l'onde sinusoïdale (KAISTRENKO et al., 1991 ; MADSEN \& FUHRMAN, 2008),

b) la linéarité : l’onde linéaire (SYNOLAKIS, 1991) et l'onde non linéaire périodique déformée (DIDENKULOVA et al., 2007) et enfin,

c) la dispersion.

Les équations de Boussinesq prennent en considération la linéarité et la dispersion, ces équations sont les plus adéquates pour être utilisées dans les zones côtières.

\section{Méthodes}

\subsection{Modèle mathématique}

Les équations de WEI et al. (1995) utilisées par COULWAVE sont retenues.

Continuité de la masse :

$\eta_{t}+\nabla M=0$ 
La connaissance de la Mer :

un vecteur du développement durable en Méditerranée

$$
\begin{gathered}
M=(h+\delta \eta)\left\{\mathbf{u}_{\alpha}+\mu^{2}\left[\frac{1}{2} z_{\alpha}^{2}-\frac{1}{6}\left(h^{2}-h \delta \eta+\delta^{2} \eta^{2}\right)\right] \nabla\left(\nabla \cdot \mathbf{u}_{\alpha}\right)\right. \\
\left.+\mu^{2}\left[z_{\alpha} \frac{1}{2}(h+\delta \eta)\right] \nabla\left[\nabla \cdot\left(h \mathbf{u}_{\alpha}\right)\right]\right\}
\end{gathered}
$$

Équation du mouvement :

$$
\begin{aligned}
& \mathbf{u}_{\alpha t}+\mathscr{\delta}\left(\mathbf{u}_{\alpha^{*}} \nabla\right) \mathbf{u}_{\alpha}+\nabla \eta+\mu^{2} \mathbf{V}_{1}+\delta \mu^{2} \mathbf{V}_{2}=O\left(\mu^{4}\right) \\
& \mathbf{V}_{1}=\frac{1}{2} z_{\alpha}^{2} \nabla\left(\nabla \cdot \mathbf{u}_{\alpha t}\right)+z_{\alpha} \nabla\left[\nabla \cdot\left(h \mathbf{u}_{\alpha t}\right)\right]-\nabla\left[\frac{1}{2}(\delta \eta)^{2} \nabla \cdot \mathbf{u}_{\alpha t}+\delta \eta \nabla \cdot\left(h \mathbf{u}_{\alpha t}\right)\right] \\
& V_{2}=\nabla\left\{\left(z_{\alpha}-\delta \eta\right)\left(\mathbf{u}_{\alpha^{*}} \nabla\right)\left[\nabla \cdot\left(h \mathbf{u}_{\alpha}\right)\right]+\frac{1}{2}\left(z_{\alpha}^{2}-\delta^{2} \eta \eta^{2}\right)\left(\mathbf{u}_{\alpha^{\prime \prime}} \nabla\right)\left(\nabla \cdot \mathbf{u}_{\alpha}\right)\right\} \\
& +\frac{1}{2} \nabla\left\{\left[\nabla \cdot\left(h \mathbf{u}_{\alpha}\right)+\mathbb{8} \eta \nabla \cdot \mathbf{u}_{c}\right]^{2}\right\}
\end{aligned}
$$

où $\delta=a_{0} / h_{0}$, est le paramètre de non-linéarité ; quand $\delta=0$ les équation deviennent linéaires, et $\mu=k_{0} h_{0}$ est le terme de dispersion, et $a_{0}$ et $h_{0}$ sont respectivement l'amplitude et la profondeur représentatives (typique).

$z_{\alpha}=\left[(1+z \alpha)^{\frac{2}{2}}-1\right] \hbar \approx-0.531 h$

$\alpha=\frac{1}{2}\left(\frac{z_{\alpha}}{h}\right)^{2}+\frac{z_{\alpha}}{\hbar} \approx-0.390$

$\mathbf{u}_{\alpha}=$ la vitesse horizontale à $z=z_{\alpha} \quad \mathbf{u}_{\alpha}=(\nabla \phi)_{z=z_{\alpha}}$

\subsection{Modélisation}

La modélisation se fera à une dimension, pour une vague non déferlante $(H / d=0.0185)$ sur une plage plane de pente $\operatorname{cotg} \beta=19.85$, la pente débute à $x / d=120$, la position initiale de la crête de la vague se trouve à $x / d=45$, au repos la ligne d'eau croise la plage au niveau 0 et à $x / d=139.85$, et $t=t^{*}(g / d)^{0.5}$, où $t^{*}=$ le temps (voir figure 1 ). Quand le fond est horizontal le nombre d'Ursell est de l'ordre de 1, mais sur la pente il devient si grand qu'on ne peut plus utiliser la théorie linéaire.

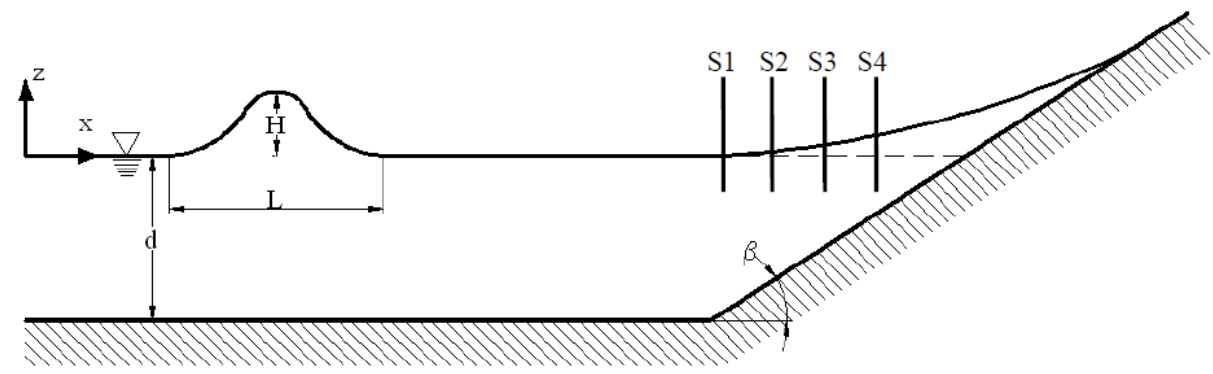

Figure 1. Schéma du canal de la modélisation

( $L=$ longueur d'onde, $H=$ amplitude, $d=$ profondeur d'eau, $\beta=$ angle d'inclinaison,

$S 1, S 2, S 3, S 4=$ sondes d'enregistrement).

\section{Résultats}

\subsection{Enregistrement des sondes}

Les séries de temps sont obtenues par l'enregistrement des sondes électriques (voir figure 2), ces sondes nous donnent la variation du niveau d'eau dans le temps dans 
l'endroit d'installation de la sonde. Nous avons pris quatre sondes installées dans quatre endroits différents (S1, S2, S3 et S4) choisis d'une façon significative pour mieux présenter les effets de la plage sur le comportement de la vague (voir figure 1). On remarque la présence de deux piques qui sont l'onde de tsunami et l'onde réfléchie par la pente, ainsi que le creux qui représente le run-down.

\subsection{Surfaces libre}

La représentation de la surface d'eau est une image instantanée prise comme coupe longitudinale du canal qui montre l'élévation du niveau d'eau le long de $x$ (figure 3). La valeur maximale adimensionnelle du run-up obtenue par simulation est égale à 0.09.

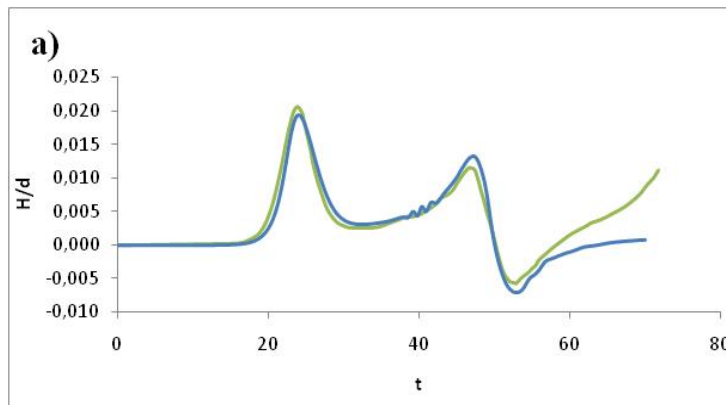

c)

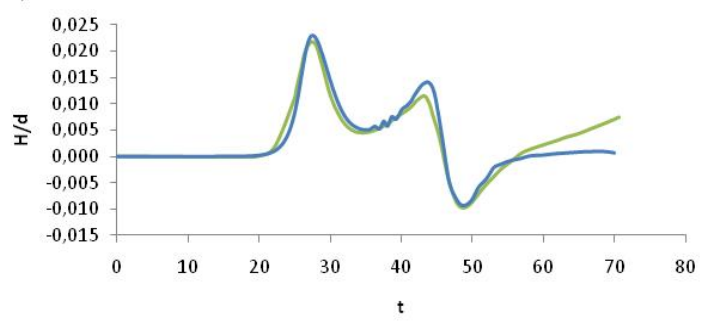

b)

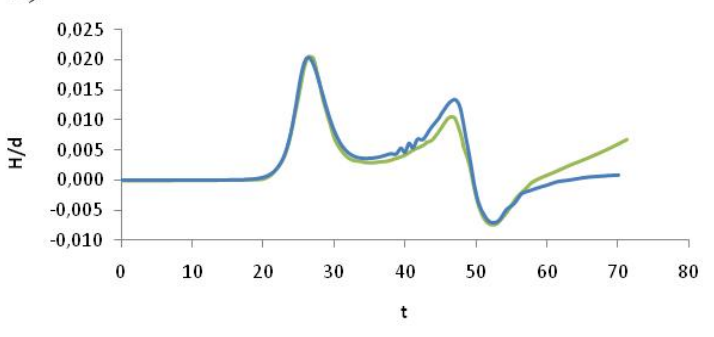

d)

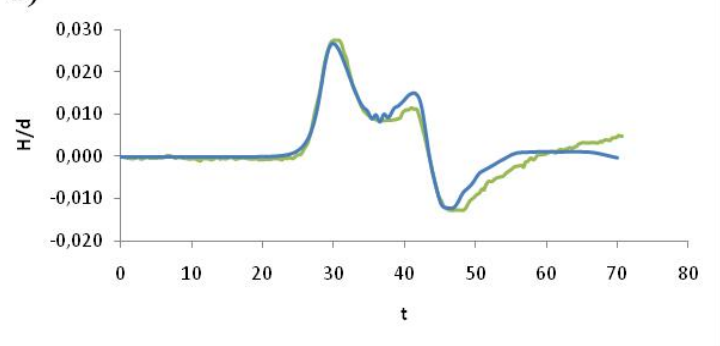

Figure 2. Enregistrement des sondes (a) S1, (b) S2, (c) S3 et (d) S4

( $a, b, c$ et $d$ sont situés respectivement à $x / d=120,124.14,129.9$, et 134.75).

Les résultats expérimentaux de SYNOLAKIS (1987) sont tracés en ligne verte.

Nos résultats numériques sont tracés en ligne bleue.

\section{Conclusion}

On voit que les séries de temps et les formes de surface libre obtenues par modélisation numérique concordent bien avec les résultats expérimentaux, d’où la fiabilité du modèle utilisé (Boussinesq) ainsi que le code de calcul (COULWAVE).

\section{Références bibliographiques}

DIDENKULOVA I., PELINOVSKY E., SOOMERE T., ZAHIBO N. (2007). Run-up of nonlinear asymmetric waves on a plane beach. In: Tsunami and Nonlinear Waves, Ed. By KUNDU A., Springer, pp 175-190. 
La connaissance de la Mer :

un vecteur du développement durable en Méditerranée
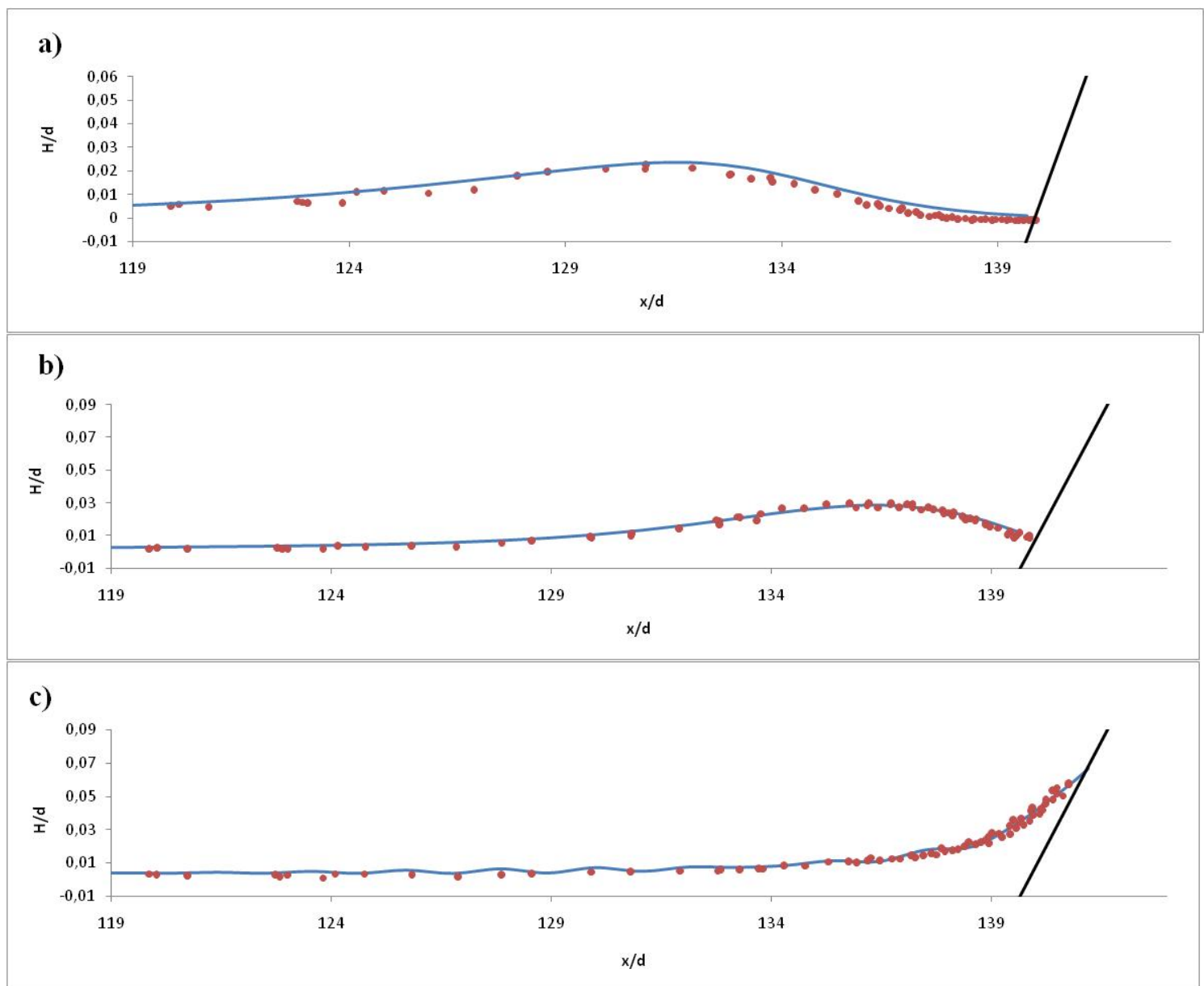

Figure 3. Surface libre à (a) $t=30$, (b) $t=33$, (c) $t=39$

(La plage est représentée par une ligne noire, résultats expérimentaux en points rouges, nos résultats numériques en ligne bleue).

KAISTRENKO V.M., MAZOVA R.K., PELINOVSKY E.N., SIMONOV K.V. (1991). Analytical theory for tsunami runup on a smooth slope. International Journal of the Tsunami Society 9, pp 115-127.

MADSEN P.A., FUHRMAN D.R. (2008). Run-up of tsunamis and periodic long waves in terms of surf-similarity, Coastal Engineering 55, pp 209-223. doi:10.1016/j.coastaleng.2007.09.007

SYNOLAKIS C.E. (1987). The run-up of solitary waves. Journal of Fluid Mechanics 185, pp 523-545. doi:10.1017/S002211208700329X

SYNOLAKIS C.E. (1991). Tsunami runup on steep slopes: How good linear theory really is. Natural Hazards 4, pp 221-234. doi:10.1007/BF00162789

WEI G, KIRBY J.T, GRILLI S.T, SUBRAMANYA R (1995). A fully nonlinear Boussinesq model for surface waves. Part 1. Highly nonlinear unsteady waves. Journal of Fluid Mechanics 294, pp 71-92. doi:10.1017/S0022112095002813 